Compter les « retours » d'émigrants dans l'Italie du début du XXe siècle : conventions statistiques, libéralisme économique et politique publique

Counting Emigrants' "Returns" to Italy at the Beginning of the 20th Century:

Statistical Conventions, Economic Liberalism and Public Policy

Contabilizar los retornos de emigrantes en Italia al comienzo del siglo XX:

convenciones estadísticas, liberalismo económico y políticas públicas

\title{
Caroline Douki
}

\section{OpenEdition}

\section{Journals}

Édition électronique

URL : https://journals.openedition.org/remi/6470

DOI : $10.4000 /$ remi.6470

ISSN : $1777-5418$

Éditeur

Université de Poitiers

Édition imprimée

Date de publication : 1 septembre 2013

Pagination : 11-32

ISBN : 979-10-90426-09-2

ISSN : 0765-0752

\section{Référence électronique}

Caroline Douki, «Compter les « retours » d'émigrants dans I'Italie du début du XXe siècle : conventions statistiques, libéralisme économique et politique publique », Revue européenne des migrations internationales [En ligne], vol. $29-n^{\circ} 3$ | 2013, mis en ligne le 01 septembre 2016, consulté le 14 avril 2022. URL : http://journals.openedition.org/remi/6470 ; DOI : https://doi.org/10.4000/remi.6470 


\section{Compter les " retours " d'émigrants dans I'Italie du début du XXe siècle : conventions statistiques, libéralisme économique et politique publique}

\section{Caroline Douki ${ }^{1}$}

Comme nombre de termes utilisés pour rendre compte du déplacement des populations migrantes dans l'espace géographique et social, celui de " retours" ne saurait être pris comme allant de soi. Pourtant, alors que des notions comme celles d' " assimilation ", " intégration ", " diaspora ", ont depuis longtemps fait l'objet d'un travail d'objectivation critique ${ }^{2}$, les " retours " sont encore souvent envisagés, lorsqu'ils sont pris comme objet historique, comme s'il s'agissait seulement d'une réalité sociale, que les appareils statistiques du passé n'auraient eu qu'à compter, le rôle de I'historien consistant alors à incarner ou expliciter les données chiffrées en reconstituant des itinéraires ou en dressant des typologies selon les raisons d'agir des migrants ${ }^{3}$. Or la notion même de " retour " est une élaboration conceptuelle qui remonte au tournant des XIXe-XXe siècles, et relève d'une double dynamique : elle est à la fois une construction des savoirs et un produit des politiques migratoires qui s'affirment alors.

Plutôt que de déplorer le caractère tardif de l'effort d'enregistrement statistique des retours, qui gênerait le travail des historiens occupés à reconstituer la réalité des mouvements migratoires du passé ${ }^{4}$, il faut considérer l'apparition de statistiques de " retours " migratoires (d'hommes ou d'argent) au tournant des XIXe-XXe siècles, à I'instar de tout instrument administratif de mesure : la statistique ne se contente jamais de compter des choses préexistantes, mais contribue elle-même à mettre en ordre le social et à faire advenir comme une réalité évidente ce que, souvent, elle constitue de toutes pièces ${ }^{5}$. Ainsi, c'est l'élaboration progressive d'une taxinomie spécifique, résultat d'un véritable travail de mise en forme institutionnelle et savante, qui fait qu'à un moment donné le vocable " retour " a fini par s'imposer pour qualifier systématiquement certains

\footnotetext{
1 Maître de conférences, Université Paris 8, Département d’histoire, IDHE (UMR 8533) ; carolinedouki@gmail.com

2 Parmi une importante bibliographie, voir Green (2002) et Dakhlia (2011).

3 C'est par exemple le parti méthodologique privilégié par Harper (2010).

4 C'est ce qui ressort, par exemple, des remarques méthodologiques de Wyman (1993: 7-9; 2005). 5 Sur la fonction d'objectivation mais aussi de catégorisation des instruments statistiques, dans une bibliographie désormais très développée, voir Porter (1995) et Desrosières (2008).
} 
types de flux collectifs et certains comportements des migrants. Ce processus est à l'œuvre dans de nombreux pays émigrateurs ou immigrateurs, qui ont chacun des raisons de porter un nouveau regard sur les flux migratoires inverses. Au demeurant, tous partagent une brutale prise de conscience de la massification et de I'accélération de la mobilité internationale des populations. C'est pour se forger de nouveaux instruments permettant d'interpréter ces changements et de gouverner cette mobilité qu'ont été élaborées de nouvelles catégories classificatoires, de nature indissociablement idéologique et savante. Parmi celles-ci, on connaît bien, désormais, l'importance des catégorisations nationales, raciales ou genrées. Mais il convient également de leur rattacher le nouveau découpage du processus migratoire en séquences bien individualisées (séparant départs, absences, retours).

Cet article propose de restituer, à partir de ce qui se déroule dans les sphères savantes et administratives italiennes au cours des années 1870-1920, le caractère graduel du processus par lequel le « retour » est devenu une catégorie statistique individualisée, en relation avec des questions cognitives, techniques et administratives. Pour les contemporains déjà, l'un des grands enjeux attachés à la présentation statistique du phénomène des retours résidait dans les possibilités d'utiliser ces chiffres en politique, au double sens du terme: dans le domaine des politiques publiques destinées à encadrer les migrations, comme dans celui des polémiques partisanes.

Centrer le regard sur la contribution des sphères administratives et savantes italiennes à la mise en forme internationale de la notion statistique de " retour " se justifie d'une double manière. D'une part parce que, principale nation émigratrice de I'époque, I'Italie se distingue par l'élaboration précoce d'un dispositif institutionnel destiné à étayer ce qui devient, dès le début du XXe siècle, une politique de protection des intérêts et des droits de ses émigrants ${ }^{6}$. Pour en accompagner la genèse, I'Italie a précocement développé un ample appareil d'enregistrement et d'analyse statistique de ces migrations (Franzina, 1980 ; Marucco, 2001). D'autre part, cette configuration italienne est d'autant plus instructive pour comprendre l'élaboration de nouvelles notions migratoires, qu'elle s'articule étroitement aux institutions et aux débats statistiques, économiques, démographiques internationaux de l'époque. Ainsi, depuis les années 1870, parallèlement à leur mobilisation pour la construction d'une statistique officielle, centralisée et professionnalisée, indispensable à un État-nation qui se veut moderne (Marucco, 1992 ; Patriarca, 1996), de grandes figures issues des milieux administratifs et savants italiens, à I'instar de Luigi Bodio, ont joué un rôle majeur dans les réseaux et les travaux de l'internationalisme statistique : ils ont apporté leur expérience et leur réflexion spécifique aux débats de l'époque, qui s'inscrivaient dans une échelle-monde et cherchaient donc déjà les meilleurs moyens pour produire des chiffres fiables et comparables en matière de migrations ${ }^{7}$.

\footnotetext{
6 Pour une démonstration plus développée, voir Douki (2006) ; sur la précocité des politiques italiennes, qui doit nécessairement s'étayer dans le comparatisme, voir Douki, Feldman et Rosental (2006).

7 Sur la concomitance de la mobilisation nationale et de l'activité internationale des grands statisticiens de la fin du XIXe siècle, voir Brian (1989 et 2002). Parmi les grandes figures de l'internationalisme statistique, se distingue d'emblée Luigi Bodio, qui a joué un rôle déterminant dans I'organisation de la statistique nationale du nouvel État italien, tout en étant secrétaire général (de 1885 à 1905) puis président (après 1909) de I'Institut International de Statistique (Soresina, 2001).
} 


\section{Première étape de la formalisation statistique : retours trop visibles ou retours impensés?}

Depuis 1876, le nouvel État italien a réussi à imposer aux acteurs économiques privés, et face aux prétentions classificatrices de certains États récepteurs, son propre appareil statistique sur les migrations : organisée pour être vraiment nationale et centralisée, la " statistique annuelle du mouvement de l'émigration italienne ", est réalisée et publiée par la Direzione Generale della Statistica (DGS) : celle-ci utilise les relais administratifs périphériques (mairies, préfectures et bientôt consulats) pour collecter l'information, en leur imposant des normes homogènes de relevé et en réservant aux seuls services centraux I'élaboration mathématique et formelle des résultats, ainsi que leur commentaire ${ }^{8}$.

Dès cette première phase, le phénomène des retours est d'une certaine façon pris en considération. Toutefois, il ne s'agit pas, alors, de les étudier en eux-mêmes : on enregistre seulement les intentions de rapatriement exprimées par les candidats au départ lorsqu'ils sollicitent auprès de leur mairie l'autorisation (nulla osta) nécessaire à la délivrance d'un passeport. Les limites de cette méthode (le caractère partiel de la source, les involutions qui peuvent se produire entre déclarations au départ et itinéraires postérieurs), seront assez rapidement soulignées par les experts de la DGS, mais au moment de la création de cet instrument, I'enjeu n'est pas là. Sur la base de ces déclarations, la statistique tente surtout de classer les expatriations en deux catégories bien distinctes : I'" émigration temporaire " (emigrazione temporanea), différenciée de I'" émigration proprement dite " (emigrazione propria), entendue comme définitive. Cette volonté de séparer, dès avant le départ, deux types de mobilité ne correspond pas à une lecture de la migration distinguant clairement les étapes d'une trajectoire, mais relève avant tout de l'effort pour avancer une lecture officielle de la nature de l'émigration massive en train de se développer dans la Péninsule. L'enjeu est fondamental pour un État récemment constitué, qui s'est donné pour mission de rassembler une société en profonde mutation : il lui faut cerner officiellement ce qu'il peut considérer comme véritable émigration (" l'émigration proprement dite "), c'est-à-dire faire la part entre ce qui relève de pratiques familières dans un pays qui, à l'instar de toutes les sociétés montagnardes ou méditerranéennes (Fontaine, 2005 ; Dakhlia, 2013), a toujours été habitué à la pluriactivité rurale et à la mobilité du travail, et les nouvelles formes de migration, inédites par leur massivité, leurs rythmes et les directions empruntées (avec le déploiement des flux outre-Atlantique).

Si la statistique nationale cherche bien à enregistrer le plus exhaustivement possible toutes les formes de mobilité, par l'effet de la nomenclature binaire utilisée, I' " émigration temporaire ", c'est-à-dire celle qui implique des retours, est officiellement donnée à lire comme n'étant pas " une émigration proprement dite ". L'objectif de ce système statistique de différenciation est d'abord politique : en mettant ainsi en évidence la grande part d'émigration temporaire dans les pratiques de mobilité des Italiens, et en la rattachant à des formes

8 Les classements sont annuels, les résultats sont publiés tous les deux ans : MAIC (Ministero dell'Agricultura, Industria e Commercio), DGS (1976-1924) Statistica della emigrazione italiana all'estero, Rome, 29 volumes. 
traditionnelles, on peut inclure ces migrants dans la description statistique d'ensemble du nouvel État-nation et leur donner une place dans sa représentation officielle, en affirmant par là même que cette population n'est pas considérée comme perdue pour le pays d'origine. On perçoit ici la stratégie d'un État récent qui entend affirmer la continuité de sa souveraineté sur la population momentanément éloignée de son territoire, le message s'adressant autant à la société italienne qu'aux pays d'immigration ${ }^{9}$. Cela a une implication importante pour notre démonstration : en soulignant ainsi la propension à revenir comme une caractéristique très répandue parmi les migrants, on distingue moins des retours (précisément conceptualisés) qu'on ne cherche à nuancer la tendance des Italiens à véritablement partir. En somme, dans cette période transitoire que sont les années 1870-1880, où la mobilité s'intensifie, dans un flou mêlant anciennes et nouvelles circulations, savants et administrateurs italiens travaillent encore prioritairement sur la notion de départ.

Cette manière d'appréhender la mobilité, qui ne déglobalise pas clairement les trajectoires et ne procède pas encore à un séquençage départ/retour, se perçoit à la base comme au sommet du processus de production des statistiques migratoires. Chargées de la première étape du travail de recueil des données, puisqu'elles sont au plus près du terrain, les administrations municipales rencontrent de nombreuses difficultés pratiques et cognitives pour relever et classer les départs (Douki, 1994). Jusqu'au milieu des années 1880 au moins, certaines ne comptabilisent pas les départs que leurs administrés annoncent limités à quelques mois de travail à l'étranger : aux yeux des édiles, encore empreints d'une vision peu administrative de la mobilité, il ne s'agit pas $d^{\prime}$ " émigration proprement dite ", et parfois pas même d'" émigration temporaire ", tant ces personnes restent, malgré I'absence, attachées à la vie locale, et tant ces mouvements sont familiers. Ces difficultés à appliquer de manière rigoureuse la taxinomie et les normes d'enregistrement élaborées par les services centraux mettent en lumière les délais qui furent nécessaires à l'acculturation aux nouvelles lectures statistiques dans les profondeurs de la société italienne, comme aux périphéries de l'État.

Mais il faut aller au-delà de cette première explication. Les réticences des maires et des services municipaux ne sont pas toujours dues, comme la DGS le leur reproche souvent, à une absence de zèle ou à la faiblesse de leurs compétences bureaucratiques ${ }^{10}$. Elles découlent également de leur position d'observation, rapprochée et profondément impliquée, qui les ancre dans une logique particulièrement signifiante pour notre démonstration. En dépit des normes qui leur sont maintes fois rappelées par circulaires, leur lecture se fonde avant tout sur une familiarité qui leur donne aussi une certaine compétence de jugement, même si celle-ci est moins technique et apparemment plus " ordinaire ${ }^{11}: c^{\prime}$ est bien parce qu'ils sont plongés dans les profondeurs du

9 Pour une démonstration plus développée, voir Douki (2006).

10 Les défaillances de l'échelon municipal dans le relevé statistique de l'émigration sont mises en relief par une enquête étatique menée en 1903 ; cf. MAIC, DGS (1906) Statistica della emigrazione italiana per I'estero negli anni 1904 e 1905, Rome, pp. V-VI.

11 Sur la notion de " jugement ordinaire " présent dans les activités de classement des agents d'observation ou d'enregistrement en situation de familiarité, voir Thévenot (2006: 141-147). 
tissu social et du fonctionnement économique rural que des responsables municipaux tiennent toujours à nuancer l'existence d'une forte émigration dans leur commune, quand ils savent, par expérience, que leurs administrés travaillant à l'étranger, bien qu'absents pendant un certain temps, ne sont pas vraiment " partis " au sens profane du terme... puisqu'ils vont revenir et se tiennent en relation constante avec leur famille, faisant vivre, par leurs revenus envoyés ou rapportés de l'étranger, l'économie domestique et l'économie locale. Jusqu'à la fin des années 1880, une partie des édiles administrant des communes où le travail à l'étranger est pourtant une réalité incontestablement importante, continuent à résister à l'idée qu'il faille parler, comme l'idiome statistique officiel le conçoit, de " départ " ou même d' "émigration ", pour cette simple raison qu'ils sont assurés du retour des personnes concernées. On arrive ainsi à ce paradoxe, qui perdure jusqu'à la fin du XIXe siècle dans le cas italien : les migrants saisonniers et ceux qui se déclarent d'emblée dans un projet temporaire n'étant pas considérés comme "émigrants proprement dits ", il est difficile de concevoir formellement pour eux la catégorie statistique de " retour ", alors même qu'ils sont précisément ceux qui reviennent massivement.

Dans ce moment de transition, un autre problème surgit bientôt. De plus en plus exhortés à remplir régulièrement les bulletins de dénombrement destinés à la DGS, de nombreux secrétaires de mairie vont procéder à des codages expéditifs, qui contribuent d'une autre manière à fausser la perception des réels mouvements de retour : pour distinguer émigration temporaire et définitive, ils retiennent le plus souvent comme critère l'éloignement de la destination (la traversée de l'Atlantique faisant office de point de repère le plus courant). Mais enregistrer systématiquement comme définitifs les départs vers l'Amérique, et comme temporaires les départs vers les pays européens ou le pourtour méditerranéen, est bien loin de correspondre à la réalité des pratiques (Cerase, 2001). Ce décalage croissant entre observations rapprochées et observations transcrites dans les codifications statistiques est propice à un double brouillage des mouvements dont on veut rendre compte, puisque se conjuguent ici le flou des classements et la réification des catégories, qui gênent beaucoup la mise en visibilité statistique des flux inverses.

Dès la fin des années 1870, en introduction de chaque volume de la statistique de l'émigration italienne, Luigi Bodio, responsable de la DGS, multiplie les avertissements sur le caractère flou ou arbitrairement tranché des données ainsi classées. De ces remarques méthodologiques, il nourrit les innombrables discussions sur la nature des migrations internationales auxquelles il prend part à l'intérieur de la sphère étatique italienne, ainsi que dans l'espace international du débat savant, notamment pendant les sessions annuelles de l'Institut International de Statistique, où statisticiens et économistes s'intéressent particulièrement à la question et le reconnaissent alors comme un expert de tout premier ordre. Ce que traduisent les tâtonnements des agents administratifs italiens sur le terrain, comme les avertissements répétés et les propositions alternatives de l'un des plus éminents statisticiens de l'époque, est bien la difficulté, pendant un certain temps, à définir ce qu'est vraiment le départ et, conjointement, à penser la notion de retour comme une séquence spécifique, au sens où on l'entend aujourd'hui - ce sens ayant précisément été construit par le débat politique, la réflexion économique et le travail statistique du début du XXe siècle. 
En attendant, en Italie, ces premières opérations statistiques que I'on destinait, dans une optique politique, à mesurer et classifier les flux de départ pour en souligner le caractère maîtrisable, produisent un effet performatif non recherché : elles surexposent des partants et des absents dans une réalité qui, au contraire, s'apparente beaucoup plus, avant la Grande Guerre, à un vaste mouvement de turn-over qu'à un déversement continu vers l'étranger. Or, même si la publication régulière des chiffres s'accompagne de commentaires signalant qu'ils ne reflètent pas toute la complexité de la mobilité, de plus en plus dans le débat public, les migrants sont représentés sous la catégorie du départ et par les images de l'exil massif, alors qu'aucun chiffre officiel ne vient, avec le même pouvoir d'imposition symbolique que l'on reconnaît aux " grands nombres " (Desrosières, 1993 ; Goody, 1979), mettre en regard la masse des retours. Dans l'arène politique, en tirent profit les nationalistes italiens, qui ne cessent, au début du XXe siècle, de dénoncer l'émigration comme un affaiblissement de la nation, infligé par un ordre international inégal qui viderait I'Italie de sa substance, la privant de ses richesses en hommes et en force de travail ${ }^{12}$.

Après 1900, la démultiplication des efforts de mise en chiffres des retours d'émigrants vers la Péninsule représente donc à la fois un tournant en termes de savoirs et une réponse à ces enjeux politiques.

\section{Nouveaux regards sur les flux inverses}

La construction de la catégorie statistique et administrative " retour " ne s'opère que progressivement, en regroupant des situations et des mouvements assez différents, qu'il n'était pas d'emblée évident de tous inscrire dans une même classe d'équivalence. Au milieu des multiples circulations inverses entre le pays de travail et la localité italienne d'origine, les discussions des experts révèlent qu'il n'allait pas de soi, à l'époque, de placer sur le même plan des phénomènes aussi divers que le va-et-vient des migrants saisonniers, un retour de plusieurs années entre deux séquences de travail à l'étranger ou un rapatriement définitif. Même flou sur la manière dont il fallait considérer les retours de ceux qui restaient domiciliés en Italie, par rapport aux brèves visites familiales effectuées par des personnes installées durablement ou définitivement à I'étranger. Et comment faire le lien entre le retour d'une main-d'œuvre circulant selon ses propres rythmes ou selon la conjoncture du marché du travail, et ce qui relève de rapatriements contraints parce qu'ils sont la conséquence d'expulsion après installation ou le fait des refoulements immédiats qui se multiplient à partir des années 1890 dans les ports d'entrée sur le continent américain (Daniels, 2004)?

Les spécialistes de la statistique ont montré que le processus de " qualification ", dimension importante de la formalisation statistique d'un fait social, qui finit par regrouper en une même classe d'équivalence des actes ou des cas divers qui auparavant n'étaient pas reliés entre eux, s'élabore à travers tout un ensemble de jugements et de négociations scientifiques ou sociales. II en va de même ici. C'est l'émergence d'une approche plus globale de l'émigration,

12 Sur les images de l'émigration dans le discours nationaliste italien, voir Grange (1994: 977-1034) ; Salvetti (1995) et Choate (2008: 147-188). 
comme phénomène social et économique lié au travail, qui va conduire à l'organisation, en Italie, d'un nouveau dispositif statistique pour mesurer les retours. Cette nouvelle manière de lire les migrations s'est décantée au fil d'intenses échanges dans plusieurs sphères situées entre savoir et pouvoir.

\section{Une nouvelle politique migratoire italienne en quête d'instruments de mesure}

II faut tout d'abord la mettre en relation avec l'engagement des pouvoirs publics dans une action volontariste d'encadrement des migrants, qui conjugue objectifs économiques et réforme sociale. À partir des années 1900, l'État italien se lance dans une politique globale qui va au-delà $d^{\prime}$ " une politique du départ " (Green et Weil, 2006), déterminé qu'il est désormais non seulement à donner aux migrants une place plus légitime et plus visible dans la construction nationale, mais aussi à faire de la mobilité internationale de sa population rurale une véritable ressource de développement économique. À cette fin, des institutions sont créées dès 1901, notamment une agence administrative centrale chargée de coordonner l'action publique, le Commissariat Général à l'Émigration (CGE). Le rassemblement et la circulation de l'information quantitative et qualitative sur tous les aspects possibles des migrations deviennent alors le moyen crucial d'une indispensable coordination, d'autant plus nécessaire que l'objectif est d'encadrer et de protéger au mieux les migrants dans l'ensemble de leur parcours migratoire.

Ce qui signifie que les pouvoirs publics italiens visent non seulement à améliorer les conditions de départ et de voyage des émigrants, à les protéger autant qu'il est possible lorsqu'ils sont à l'étranger, mais aussi qu'ils se soucient beaucoup des retours. Notamment parce que ceux-ci représentent un enjeu économique majeur pour les familles concernées et pour l'économie italienne elle-même. C'est dans cette optique qu'apparaissent des mesures destinées à favoriser le rapatriement des revenus du travail à l'étranger, les remises (Balletta, 1976 ; Mittone, 1984 ; Douki, 2000 ; Massulo, 2001), mais également des instruments de gouvernement, de nature juridique, économique, financière et sociale, destinés à lever le plus possible d'obstacles au rapatriement des migrants, voire à faciliter la pratique des retours fréquents (Douki, 2013).

On comprend donc pourquoi le séquençage du processus migratoire devient un élément important de recomposition de l'action publique. II s'agit désormais de disposer de données suffisamment nombreuses et variées pour développer le savoir sur toutes les étapes de la migration et particulièrement celle qui est la moins connue : le retour. Dans ce nouveau contexte, l'observation des retours se trouve donc dégagée des enjeux rappelés plus haut, pour faire l'objet d'une construction statistique spécifique ayant vocation, cette fois, non plus seulement à représenter l'État-nation mais à fournir des données formalisées pour penser et administrer une question de société.

Pour que prenne forme la catégorie administrative et statistique de " retour ", il a fallu que soit élaborée dans de nombreux échanges scientifiques, puis consolidée (par son inscription dans la loi d'encadrement de l'émigration de 1901) une nouvelle représentation plus globale de la mobilité transnationale des Italiens, envisagée non plus à travers les paradigmes de l'exil et de l'arrachement (qui conduisent à privilégier l'observation du départ et de la distance spatiale), mais 
à travers le fonctionnement de l'émigration comme une circulation économique, une circulation de main-d'œuvre. C'est, en effet, lorsqu'on indexe la définition de l'émigration sur sa fonction (le travail et le rassemblement de revenus à travers les frontières), et non plus sur l'exode et la distance, qu'on peut envisager le fait migratoire dans sa globalité avec sa trajectoire d'ensemble, subdivisée en plusieurs séquences (départ, absence, retour), le tout selon des temporalités variables. Ce qui débouche, en Italie, sur la construction d'une catégorie administrative et statistique de "retour", englobant tous les cas, quels qu'en soient les rythmes : va-et-vient saisonniers, retours périodiques mais espacés, retours improvisés liés aux soubresauts de la conjoncture économique ou de la politique internationale, rapatriements définitifs liés à la fin d'un cycle de travail ou à l'âge, etc.

\section{Reconfigurations internationales et nationales des savoirs démographiques et économiques}

La catégorie de " retour " n'est pas qu'une construction administrative liée aux objectifs des pouvoirs publics, elle est aussi impulsée par divers champs du savoir qui, entre XIXe et XXe siècles, sont en train de devenir, par un biais ou un autre, des sciences de la population : économie, psychologie, anthropologie, hygiène et médecine, démographie, statistique, etc. À I'heure où ces diverses sciences sont en lutte pour leur institutionnalisation et leur développement, certaines d'entre elles vont plus particulièrement prendre en charge l'objet migration et contribuer à en rénover la compréhension comme un processus complexe et dynamique, non linéaire, dont non seulement les causes mais aussi les effets directs et indirects doivent être évalués et, dès lors, faire l'objet de nouveaux investissements d'observation et de mesure. Ces débats s'entendent à l'échelle internationale.

Les effets de l'émigration attirent évidemment l'attention de ceux qui s'emploient à approfondir les théories scientifiques, de type démographique, sur la population. Tout au long du XIXe siècle, au fil des controverses ouvertes dans le sillage des propositions de Malthus, les manières de concevoir les lois d'évolution des populations et les interactions entre populations, ressources naturelles ou économiques avaient beaucoup évolué. En décalage avec l'argumentaire malthusien classique qui voyait dans la surpopulation la cause de l'émigration, dans la seconde moitié du XIXe siècle, notamment à cause du cas de I'Irlande et du fait de la transformation des rapports villes-campagnes en Europe, c'est bien plus souvent le lien entre émigration et dépeuplement qui se retrouve au centre des interrogations. Au tournant des XIXe-XXe siècles, ces questionnements ont fini par produire des argumentaires très contrastés : dans ces dissonances, avancer de nouvelles formules de chiffres devient un enjeu majeur. À cette époque ces interrogations sont déjà posées de manière comparative, au moins entre pays européens, et les savants italiens, très présents dans ces débats, contribuent à les faire rapidement évoluer en recherchant de nouveaux types de mesures des effets démographiques de l'émigration. Certains d'entre eux, à l'instar de Francesco Coletti (universitaire reconnu pour ses compétences statistiques et démographiques, qui s'intéresse aux mutations du monde rural italien provoqué par l'émigration), rejoignent Bodio sur la nécessité de cerner plus rigoureusement le caractère non définitif d'une large partie de l'émigration, et appellent donc à un perfectionnement des instruments de mesure des rapa- 
triements ${ }^{13}$. Ils veulent notamment obtenir un ratio départs/retours le plus précis possible, dans le but de produire une mesure qui puisse permettre d'évaluer au plus juste les soustractions de masses de population.

La science économique, dont les questionnements et les méthodes sont alors en renouvellement, notamment autour de la mathématisation des modes de raisonnement, contribue, plus encore que la démographie, à attirer l'attention sur la nécessité d'une formulation métrologique systématique des flux migratoires inverses. Ici, à la différence des approches démographiques, qui souhaitent de nouvelles mesures pour nuancer la description de la population, c'est parce que I'on considère les mouvements migratoires (et plus généralement l'évolution de la population) comme une variable de l'économie, que l'on veut développer de telles statistiques.

Cette quête particulière est liée aux débats se déroulant, à l'échelle internationale, parmi les économistes libéraux qui s'efforcent de mettre à jour certaines des grandes questions du schéma libéral classique : la mesure de la richesse d'une nation et celle des échanges économiques entre les pays. On veut, en effet, dépasser les outils utilisés depuis Adam Smith, ainsi que l'ancienne conception de la balance du commerce hérité du mercantilisme, pour tenir compte des mutations qui touchent la sphère des échanges économiques internationaux désormais de plus en plus nombreux, variés et rapides. L'enjeu (qui est, par exemple, clairement explicité dans ce forum de débats et de coordination méthodologique qu'est alors, pour les penseurs économiques libéraux, I'Institut International de Statistique), est de parvenir à une reformulation théorique et statistique de la "richesse des pays ", ou de la " richesse nationale ", en y introduisant, comme composante à part entière, la notion plus large de "balance économique internationale ", qui prenne en compte non seulement les échanges de marchandises, mais aussi toutes les autres formes d'échanges ayant valeur économique, comme la circulation internationale de numéraires, de titres, de crédit et $d^{\prime}$ hommes $^{14}$. Parce qu'elles font circuler de la force de travail et des revenus, les migrations sont identifiées, dans ces débats, comme l'un des phénomènes récents et massifs qui participent activement de l'intensification des échanges économiques internationaux : il devient donc urgent de se doter de nouveaux instruments permettant d'en mesurer au plus juste les quantités; et cela dans tous les sens de circulation, à l'aller comme au retour. La perspective finale étant de tenter d'en calculer la valeur ${ }^{15}$, pour la mettre en compte dans la balance économique des pays d'origine ou dans celle des pays qui emploient des étrangers. Dans cette perspective les besoins de statistiques visent à la fois les flux de personnes et les flux financiers qui les

13 Coletti Francesco (1911) Dell'emigrazione italiana, Milan, Hoepli. Professeur aux universités de Pavie et Milan au début du XXe siècle, il est également membre du Conseil Supérieur de la Statistique entre 1910 et 1926.

14 Ces questions sont envisagées dans la pensée économique libérale depuis les années 1870. Débattues en termes théoriques, et autour d'une formalisation statistique dans les travaux de la section économique de I'Institut International de Statistique [IIS] fondé en 1885, elles y sont sérieusement approfondies après 1905. Les termes placés entre guillemets dans cet article sont les appellations proposées lors des débats aux sessions de Londres (1905), Copenhague (1907), La Haye (1911). Sur I'IIS et les économistes, voir Gagnon (2000: 206-207).

15 Sur la distinction des deux opérations techniques et cognitives, mesurer/évaluer, voir Vatin (2009). 
accompagnent (les remises renvoyées périodiquement ou les revenus rapportés au moment du retour).

Ces enjeux qui relèvent de la sophistication du modèle économique libéral lui-même et de son schéma de l'équilibre général, ainsi que les complexes débats métrologiques qui leur sont liés (autour de la technique statistique et du renouvellement possible ou non des formules mathématiques à mobiliser), sont donc de plus en plus débattus à l'échelle internationale, avec d'importantes contributions des experts italiens, notamment Luigi Bodio très actif dans les efforts de formalisation déployés à I'IIS. Certains d'entre eux font aussi l'objet d'une controverse dans l'espace scientifique italien. L'action coordinatrice du tout nouveau CGE provoquant une amplification de l'effort d'évaluation des flux, et annonçant, en même temps, la volonté de placer l'émigration en bonne place dans la politique de développement économique du pays, les années 1900 et 1910 sont donc le moment choisi par des économistes, plus aguerris que la génération précédente au maniement de nouveaux outils mathématiques, pour donner une résonnance proprement nationale, à tous ces questionnements conceptuels ou comptables sur la profitabilité de l'émigration pour le pays d'origine. Ils utilisent pour cela les plus grandes revues italiennes d'économie, notamment le Giornale degli economisti qui, après avoir été un organe de restructuration de la pensée économique libérale dans I'Italie unifiée, notamment autour de l'économie mathématique, est devenu un vecteur de diffusion des nouvelles idées en matière de finances publiques ${ }^{16}$. La discussion des propositions tourne rapidement à la controverse savante et aux luttes de positionnement académique, mais il est un point sur lequel les diverses parties sont en accord : la nécessité de fonder une statistique des mouvements de retours (ceux des hommes et ceux des revenus), condition sine qua non pour nourrir de véritables tentatives d'évaluation du rapport coût/profits de l'émigration.

Parce que ces débats se déroulent autant à l'échelle du contexte scientifique international qu'à l'échelle nationale des pays les plus concernés par la question, on ne s'étonnera pas d'y voir déjà poindre la recherche de formules communes de classification et de mesures statistiques de ces flux. On en trouve trace dans les débats de I'IIS, où l'on pense bien sûr à la comparaison entre pays. Certains y évoquent aussi la nécessité de ne pas seulement mettre en regard les données des différents pays concernés, mais de réfléchir à une manière de véritablement les croiser. On sait, en effet, que si les rencontres savantes internationales étaient incontestablement l'occasion d'exprimer, voire d'attiser, des concurrences nationales (Feuerhahn, 2010), certains forums comme I'IIS ont également permis l'avancée d'un processus de partage international et d'universalisation de I'approche statistique (Gagnon, 2000 ; Brian, 2002). Toutefois, la prise en compte de normes internationales de classification et d'évaluation des flux de va-et-vient d'hommes et de capitaux liés aux migrations de travail reste en suspens avant 1914. Pour deux raisons. D'une part les appareils de relevés statistiques des migrations, lorsqu'ils existent, restent nationaux, avec leur méthodologie propre, que l'élaboration théorique, même partagée à l'échelle

16 Une série d'articles signés par Alberto Beneduce, alors en position de jeune franctireur, puis Francesco Coletti et Vilfredo Pareto, se répondant et se critiquant les uns les autres, est publiée en rafale dans le Giornale degli Economisti entre décembre 1904 et août 1905. La controverse reprend ponctuellement en 1910 et 1912. 
internationale, ne peut à elle seule rapidement faire changer. D'autre part, dans les pays les plus précocement mobilisés sur ce front, à l'instar de l'Italie, l'organisation du dispositif de relevé, de classification et de calcul des flux de retours a rencontré de très nombreuses difficultés.

\section{Structures et apories de la statistique italienne des retours}

Le réagencement de la statistique italienne de l'émigration s'est opéré par ajustements successifs ${ }^{17}$. Parmi les principales modifications figure l'abandon, en 1902, de l'impossible distinction, préalable au départ, entre " émigration temporaire " et " émigration proprement dite " ou définitive, remplacée par l'établissement de deux séries de chiffres, désormais distinctes, avec d'un côté les départs et de l'autre les retours. Et pour chacune de ces comptabilités, on a cherché à améliorer les sources d'observation ainsi que les agents chargés du relevé et des premiers codages. Le décompte des départs est retiré, en 1904, aux services municipaux et confié aux services locaux de police qui administrent la nouvelle source d'observation : les registres de passeports, qui semblent plus proches du mouvement réel que les registres municipaux de nulla osta ${ }^{18}$. Dans le contexte de l'Italie giolittienne, cette réforme ne doit pas être interprétée comme l'évolution vers une logique de police, car sa problématique est autre, professionnelle et technique : on vise à travailler avec une source-papier plus proche des mouvements réels d'expatriation et à s'appuyer sur un personnel étatique mieux aguerri à ce type de décompte complexe que ne le sont les secrétaires municipaux des communes rurales. Cela correspond, en outre, à une transformation de la police italienne dans le sens d'une meilleure formation professionnelle et bureaucratique, parce qu'on la veut plus efficace bien sûr, notamment en matière d'identification, mais tout autant pour qu'elle soit mieux insérée dans les perspectives de réformes et de démocratisation de la vie publique recherchée par l'État à cette époque ${ }^{19}$.

Par contraste avec le resserrement technique de l'observation des départs, le décompte des retours se retrouve partagé entre plusieurs points et agents d'observation. C'est que les trajectoires de retour sont beaucoup plus difficiles à repérer étant donné leur diffraction dans l'espace, et également parce qu'on tâtonne encore pour préciser la catégorie statistique. Trouver le meilleur point d'observation pour procéder au dénombrement et au premier codage dépend de ce qu'on veut étudier, à quel moment précis et dans quel but. La question est donc à la fois technique, politique et scientifique. Veut-on avant tout comptabiliser les retours dans les frontières territoriales (I'enjeu étant là d'observer le

17 CGE (1926) Annuario statistico della emigrazione italiana dal 1876 al 1925, Rome, pp. XVII-XXI.

18 MAIC, DGS (1926) Statistica della emigrazione italiana per l'estero negli anni 1904 e 1905, Rome, p. VI.

19 II faut souligner cette mutation de la police italienne à l'époque giolittienne, précocement démontrée par Jensen (1989) et sur laquelle on trouve des éléments supplémentaires dans Garnieri (1995) ou Tossati (2009). Car les démonstrations de ces chercheurs ne doivent pas être recouvertes, notamment pour un lectorat francophone, par les analyses qui, dernièrement, ont choisi de n'aborder qu'une seule face - réelle mais trop partielle de l'engagement du travail policier italien, dans les années 1900-1910, dans les techniques scientifiques d'identification aux fins d'accroissement des contrôles. 
nombre de ceux qui viennent se replacer dans la pleine souveraineté italienne ou dans son espace économique) ? La réponse s'obtiendra en comptant ceux qui repassent les frontières italiennes, c'est-à-dire en prenant comme point d'observation le point de passage des rapatriés. Veut-on observer le retour à son point final d'arrivée en Italie (l'optique étant ici à la fois politique, économique mais aussi de connaissance démographique) ? C'est ailleurs et à un autre moment, plus tardif dans l'itinéraire de retour, qu'interviendra le décompte.

La question du point d'observation implique aussi celle des instruments administratifs qui serviront de source pour les relevés et, de manière indissociable, des agents qui les effectueront. À l'époque, dans l'ensemble des administrations statistiques et à l'échelle internationale, ceux que l'on utilise pour décompter les mouvements de personnes sont de trois types : les passeports dont les mouvements sont sous le regard de la police et peuvent être observés au départ, au passage des frontières comme à l'arrivée ; les registres de population et de changements de résidence tenus par les services municipaux qui permettent de placer l'observation au point de départ et au point final de retour des rapatriés ; les documents comptables des grandes entreprises de transport international, notamment les compagnies maritimes qui, pour les besoins de leur activité commerciale, mettent en listes et en tableaux comptables des masses de données sur les mouvements des migrants qu'elles transportent, à I'aller, au retour, en transit, et que les États peuvent solliciter.

Pour établir sa statistique des retours, I'Italie écarte, à cette époque, le binôme passeport-police : dans une perspective de facilitation des retours qui articule l'attente de retombées économiques à des principes de défense de la liberté de circulation pour travailler, l'effort statistique est conçu comme un instrument de connaissance étatique mais libéral, et ne peut donc être envisagé au prix d'un contrôle tous azimuts par les instruments et le personnel policiers. II n'est donc pas question d'instaurer un contrôle supplémentaire des passeports au point d'arrivée ${ }^{20}$. La statistique italienne des retours va donc se fonder sur les deux autres types de sources disponibles.

La Direction Générale de la Statistique qui, en principe, a le monopole de la production du chiffre officiel sur l'émigration, organise donc en 1904 des procédures pour recueillir des éléments sur les retours en utilisant l'une de ses principales chaines de production de données : les registres de population et les registres de changement de résidence (anagrafe) tenus par les services municipaux. Au point final d'arrivée des rapatriés, c'est bien la seule source qui puisse être utilisée, puisque les retours ne font alors l'objet d'aucun contrôle policier en leur point final, tandis que dans la commune où ils se réinstallent s'exerce sur eux un regard municipal, pour la tenue de ces registres nécessaires à l'administration locale. Ici, on choisit de faire de la commune de départ le cadre a priori de repérage des rapatriements, sans l'interroger comme une variable éventuelle.

Pour cette nouvelle série statistique, il faut, encore une fois, faire avec l'efficacité très variable des administrations locales : carences en personnel, organisa-

20 Bodio explicite cette position italienne lors de la huitième session de I'IIS en 1901 à Budapest; cf. Bulletin de I'IIS, XIII (1). Dans la même perspective, c'est I'époque où les lois militaires elles-mêmes allègent les contraintes de conscription pour les rapatriés (Douki, 2013). 
tion matérielle défaillante, compétences très inégales des employés municipaux malgré le mouvement progressif de professionnalisation qui les gagne eux aussi (Romanelli, 1989 ; Melis et Varni, 1999 ; Ferretti, 2003), sont encore choses courantes au début du XXe siècle dans nombre de régions de la Péninsule, notamment dans les communes montagnardes ou méridionales (Douki, 1994 ; Musi, 1998). Cela pose d'autant plus problème dans le cas de cette nouvelle statistique des retours, que celle-ci exige une organisation fine des procédures et une régularité exemplaire dans la mise à jour des registres, au fil des départs et des retours qui s'accélèrent. Dans cette perspective, les instances centrales et de plus en plus professionnalisées, auxquelles reviennent traitement des données et utilisation des résultats obtenus (la DGS et le CGE), relayées par les préfets, ont multiplié les circulaires pour codifier en détail la transcription des informations dans les registres municipaux et pour préciser les procédures de recueil des données à leur transmettre. Elles ont aussi mobilisé de nouveaux supports documentaires et des procédés matériels destinés à faciliter le travail de collecte et de classement, tout en imposant une nouvelle discipline de pensée, notamment des formulaires pré-imprimés ${ }^{21}$. Mais face à d'aussi fortes attentes, les pratiques du personnel municipal rural ne pouvaient changer assez vite, et leurs défaillances dans le relevé des mouvements de retours sont de plus en plus bruyamment déplorées dans les années $1910^{22}$. C'est pourquoi, lorsque les responsables italiens discutent à I'IIS pour déterminer quels documents et méthodes de travail statistique sont les mieux adaptés, ils se trouvent souvent en décalage avec leurs homologues européens qui, à l'instar des Belges, des Autrichiens ou des Allemands, ayant comme référence les dispositifs performants des services statistiques des grandes villes de leur pays ou le Bureau prussien de statistique, défendent la supériorité de la formule fondée sur les registres municipaux de population ${ }^{23}$. Ainsi, bien qu'on commence à partager théories et méthodes d'approche des retours à l'échelle internationale, il n'est pas de solution qui puisse alors s'appliquer communément à tous les cas, notamment du fait des discordances documentaires et organisationnelles entre pays ruraux ou pays urbanisés, et selon les régimes d'articulation entre administrations centrales et administrations locales propres à chaque nation.

Cela contribue à expliquer que c'est finalement un autre indicateur, construit autour du transport maritime, qui constitue l'axe majeur de la quantification des retours en Italie avant 1914 et que le CGE lui-même s'en fait le coordinateur. Dès 1904, cette agence gouvernementale, grande utilisatrice des statistiques de la DGS, devient elle aussi, au motif de l'urgence de ses besoins, productrice de statistiques dans les domaines les plus neufs de son champ d'action. C'est le début d'un effort mené sans relâche jusqu'aux années 1920 pour s'imposer comme un acteur central en matière de savoir sur les migrations internationales. Et c'est donc sur la question des retours, cruciale en politique et en économie,

\footnotetext{
21 Sur la diffusion de nouveaux supports écrits et procédés graphiques pour rationaliser le recueil de données chiffrées de plus en plus massives et complexes, touchant à cette époque l'ensemble des administrations privées ou publiques occidentales, voir Gardey (2008: 228-232).

22 CGE (1926) ibid., p. XVIII ; Coletti Francesco (1911) ibid.

$23 C^{\prime}$ est l'objet d'échanges animés lors des sessions de I'IIS en 1901 à Budapest et 1903 à Berlin ; cf. Bulletin de I'IIS, XIII (1) et XIV (1). Sur I'expertise technique et les schémas politiques des statisticiens allemands, voir Zimmermann (1994) et Labbé (2003).
} 
que le CGE va lancer sa première grande entreprise de savoir, en établissant sa propre statistique des retours d'Italiens en provenance d'Amérique.

Le point fort du CGE est certes de disposer d'un personnel bien formé présent sur le terrain en des points cruciaux de passage des rapatriés (dans les ports de débarquement avec ses inspecteurs d'émigration ; sur les navires avec ses commissaires-médecins de bord), mais ceux-là ne sont qu'un maillon de la chaine de production des chiffres de retours. En fait, le grand avantage du CGE est de pouvoir utiliser directement les capacités comptables des compagnies maritimes privées qui relient l'Amérique aux ports de la péninsule. Depuis 1901, la législation italienne protégeant les migrants a permis de faire de leur transport transatlantique un marché régulé, où toute compagnie maritime autorisée à y participer doit en échange respecter un cahier des charges précis, qui prévoit, outre des normes de sécurité et de confort minimal pour les migrants, des obligations de coopération avec les pouvoirs publics : les deux principales sont de laisser agir à bord un fonctionnaire italien, le commissaire-médecin, et de prendre à leur charge (en le faisant donc effectuer à leur frais et par leur personnel privé) divers contrôles et relevés nominatifs ou statistiques dont les pouvoirs publics (et notamment le CGE) ont besoin. On peut interpréter cela comme une forme de délégation de contrôle public, qui représente d'ailleurs un coût d'organisation (en temps, en personnel, en procédures bureaucratiques) non négligeable pour ces entreprises, en échange de l'autorisation d'accéder au très fructueux marché du transport maritime des travailleurs italiens, dont les va-et-vient à travers l'Atlantique, sont alors exponentiels ${ }^{24}$. C'est pourquoi, il est assez facile au CGE d'organiser son propre dénombrement systématique des retours d'Amérique. Début 1901, il dispose de données sommaires compilées par ses inspecteurs d'émigration en poste à Gênes, Naples, Palerme, Messine, à partir des listes que leur remettent les capitaines des navires. Le travail classificatoire se limite alors à distinguer les passagers selon la nationalité et la classe de voyage, puis, pour les Italiens voyageant en troisième classe (ceux qui constituent, dans la taxinomie administrative, les émigrés du travail), on procède à un classement supplémentaire par pays de provenance. À partir de 1904, alors que de son côté la DGS s'efforce de réunir des chiffres au point final de retour dans les communes italiennes, le CGE passe à la vitesse supérieure dans ses relevés aux points de passage (navires et ports) des rapatriés, en multipliant les catégories classificatoires. En 1910, pour remédier à l'inégale qualité des données fournies par les capitaines de navire, il leur impose des formulaires normalisés : ceux-ci doivent y répartir les émigrants selon l'âge, le sexe, le retour seul ou en famille, la profession, le lieu précis de provenance (pays et localité), la commune de destination en Italie, la durée de séjour à l'étranger, le caractère définitif ou seulement temporaire du retour ${ }^{25}$. Le CGE améliore donc la procédure de relevé et resserre le contrôle sur son déroulement, tout en continuant de déléguer aux compagnies maritimes la première étape du travail de compilation statistique des retours. II obtient ainsi des données beaucoup plus diversifiées sur lesquelles travailler, et plus proches des mouvements réels des migrants, puisqu'à la différence des relevés imparfaits des municipalités sur les

24 Sur l'organisation d'un marché régulé du transport transatlantique des migrants italiens, avec toutes ces implications normatives et pour la présentation du personnel du CGE présent dans les ports ou sur les navires, voir Douki (2011).

25 CGE (1911) Bollettino dell'Emigrazione, 11, pp. 9-11. 
registres de population, les transporteurs dénombrent directement, et in situ, grâce au passage par le navire qui offre une unité de lieu et de temps facilement contrôlable, des personnes se déplaçant effectivement. Le CGE est toutefois conscient que, malgré les efforts continus de perfectionnement, cette comptabilité reste partielle puisqu'elle exclut les rapatriés, sans doute assez nombreux, qui ont d'abord débarqué dans un port français, belge ou allemand, avant de rejoindre la Péninsule en train. Dans ce cas, à l'exception d'une seule entreprise (la Compagnie Générale Transatlantique, débarquant ses clients au Havre, et accréditée par l'État italien), les transporteurs ou les autorités portuaires ne sont nullement tenus de transmettre des informations aux autorités italiennes.

C'est bien l'un des points faibles du dispositif italien de décompte des retours : outre le problème posé par leur multiplicité, les points de passage n'offrent pas tous les mêmes possibilités de regard à l'administration italienne. Si sa capacité d'observation statistique est réelle dans les communes de retour et sur les navires qui débarquent leurs passagers dans les ports de la péninsule, beaucoup d'autres points de passage demeurent, par statut, dans une situation de sous-exposition statistique. Le cas problématique des ports étrangers fausse donc la mesure des retours transatlantiques établie par le CGE. Et surtout cela concerne les passages par les frontières terrestres qui, avant la Grande Guerre, ne sont pas systématiquement soumis au crible des autorités administratives. Se perpétue donc ce paradoxe : une part très importante des Italiens regagnant souvent et facilement la Péninsule, après avoir travaillé en Europe, échappe toujours à la statistique officielle des retours. Pour tous ceux qui rentrent par les ports étrangers et les frontières terrestres, l'administration et la statistique italiennes doivent donc se contenter d'indications de tendances, fournies par exemple, mais plus à titre illustratif qu'en fonction de dénombrements rigoureux, dans les rapports consulaires ${ }^{26}$.

Pour compenser, le CGE déploie une grande activité de compilation et de rapprochement de toutes les séries de données disponibles. II recherche une complémentarité entre sa série de chiffres sur les retours transatlantiques relevés au point intermédiaire de passage dans la péninsule (le navire et le port), et les séries produites par la DGS par l'observation du point final de rapatriement (la commune). II met également un grand soin à extraire ce qu'il peut utiliser parmi les statistiques migratoires rendues publiques par les États tiers qui, à l'instar des États-Unis, élaborent et publient diverses séries relatives au rapatriement, notamment avec les statistiques de refoulement au débarquement dans les ports atlantiques ${ }^{27}$. Mais les discordances dans la construction des données, comme la diversité des objectifs des administrations qui les organisent, sont difficilement réductibles. Et malgré tous les efforts des experts en chiffres du CGE, recrutés parmi la jeune génération des statisticiens rompus au renouveau mathématique de la discipline ${ }^{28}$, ces séries ne peuvent pas toutes être véritablement croisées

\footnotetext{
26 CGE, MAE (1903) Emigrazione e colonie. Raccolta di rapporti degli Agenti diplomatici e consolari, Rome.

27 Celles-ci sont livrées dans le rapport annuel de l'administration fédérale pour l'immigration ; les données concernant les Italiens en sont extraites et ajoutées en annexe des élaborations statistiques du CGE ; par exemple, CGE (1911) Bollettino dell'Emigrazione, 11 ; CGE (1926) Annuario statistico della emigrazione italiana dal 1876 al 1925; CGE (1926) L'emigrazione italiana dal 1910 al 1923, 1, pp. 40-41.

28 Sur cette nouvelle génération, voir Prévost (2009).
} 
entre elles. Or, cela seulement permettrait d'aller vers des analyses approfondies de l'ensemble des retours à l'échelle élargie de tous les flux concernant le pays, dont la caractéristique dans les années 1900-1910 est de concerner la totalité de la péninsule et de provenir d'un espace migratoire qui s'étend à très vaste échelle, entre Europe, Méditerranée et Amérique. Les avancées sont certes considérables en quelques années : dès 1911-1913, le CGE réussit à développer des élaborations statistiques très soignées des caractéristiques démographiques et spatiales des mouvements de retour, et parvient à calculer des taux de retour, des durées moyennes de séjour, ce qui permet de commencer à travailler sur le repérage des cycles mensuels ou annuels de rapatriement. Mais cela ne peut être réalisé que pour les flux en provenance d'Amérique, et au prix du travail acharné du plus brillant des experts statisticiens à disposition du CGE dans ces années, Alberto Beneduce qui s'était déjà fait remarquer pour sa participation aux controverses savantes sur les retombées économiques de l'émigration ${ }^{29}$.

Ainsi, tout en étant particulièrement avancée dans l'établissement et l'utilisation d'une statistique des retours migratoires, notamment par comparaison avec d'autres pays à la même époque, l'administration italienne fait l'expérience des difficultés spécifiques de ce type d'entreprise savante. Contrairement aux départs qui, moyennant une bonne organisation, peuvent en théorie être dénombrés à partir d'un espace principiel (la commune d'origine) toujours placé sous le regard de l'administration, pour les retours c'est la localisation même du point d'observation, qui continue de poser un problème majeur jusqu'aux années 1920. Une partie des causes du problème sont propres à I'ltalie : certaines découlent des pratiques sociales des migrants ou de l'économie des migrations (l'échelle quasi-planétaire du champ migratoire italien au début du $X X e$ siècle, la diffraction dans l'espace national et extranational des points de passage pour rentrer); d'autres relèvent de la vie des institutions ou des choix de politiques (les caractéristiques du travail administratif et statistique aux échelons périphériques de l'État et dans les municipalités rurales, le choix de ne pas instituer de contrôle policier systématique des passeports ou de déclaration obligatoire au retour). Aussi le problème du repérage et du relevé des retours ne peut-il se résoudre tant que l'administration italienne n'a pas trouvé de solution technique, ou institutionnelle, pour améliorer sa visibilité sur la totalité des rapatriés au moment où ils franchissent ses frontières, que celles-ci soient maritimes ou terrestres.

Le réaménagement du dispositif italien en 1919 est pensé pour lever une partie des difficultés : aux deux séries de dénombrement des retours créées avant-guerre, on ajoute un troisième et ultime système d'observation et de décompte, que l'on fonde cette fois sur le passeport. Celui-ci, valable trois ans, contient désormais deux feuillets détachables en cas de rapatriement(s), où

\footnotetext{
29 Beneduce Alberto (1911) Saggio di statistica dei rimpatriati dalle Americhe, in CGE, Bollettino dell'Emigrazione, 11. Avant de devenir un célèbre homme d'État, à la tête des nouvelles structures de l'intervention publique sociale et économique créées avant et pendant le fascisme, Beneduce (1877-1944) a donc commencé sa carrière comme expert mathématicien, apprécié pour ses capacités d'application des statistiques et des méthodes actuarielles aux domaines de la démographie, de l'économie et des assurances sociales, qu'il exerce dans les bureaux de la DGS à partir de 1904 puis, dans les années 1909-1911, au service du CGE. Pour ses débuts de carrière, voir Bonelli (1984). Pour une analyse plus détaillée des usages savants et administratifs des statistiques de retours transatlantiques, voir Douki (2013).
} 
sont rappelés l'état civil du migrant, sa commune d'origine (toujours postulée identique à celle du retour), le pays de provenance. Ces feuillets doivent être prélevés par les agents de police à tout passage de la frontière italienne, que celle-ci soit terrestre ou maritime. On organise donc désormais la statistique du retour, sur la base d'un contrôle de papiers d'identification et de circulation, selon un système pensé pour combiner (grâce aux feuillets renseignés) l'observation au point de passage (la frontière) retenu comme le plus pratique techniquement, ainsi que pour sa signification en termes de souveraineté, et l'indication du point final de retour (la commune). Ce nouveau dispositif de décompte fondé sur le binôme passeport-police est possible au début des années 1920 car, comme I'historiographie l'a amplement montré (Torpey, 2005), la plupart des sociétés occidentales, et en tout cas la plupart des pays fréquentés par les migrants italiens, à commencer par leur pays d'origine et de retour, ont achevé leur processus de " passeportisation " à l'occasion de la Grande Guerre et de ses contraintes spécifiques. Mais une fois la guerre achevée, les normes persistent. La solution de relevé statistique des retours choisi en 1919 par I'Italie est donc à la fois technique et politique. Techniquement, elle doit permettre de décompter tous les rapatriements qu'ils proviennent de, ou passent par l'Europe, I'Amérique, la Méditerranée. En termes politiques elle s'insère dans le contexte plus général de passage de la mobilité internationale du travail dans un nouveau paradigme de contrôle administratif presque permanent et assumé comme tel par tous les États, y compris l'État italien encore libéral à cette date, mais pour très peu de temps.

La réforme de 1919 apparaît donc comme un tournant dans I'histoire de la statistique migratoire italienne. Par contraste, on perçoit la spécificité de la période précédant la Grande Guerre, qui a vu l'élaboration théorique de la notion de retour et l'organisation des premiers dispositifs de relevés systématiques. Entre 1890-1914, en Italie comme dans les débats des milieux savants internationaux, le paradigme de connaissance et d'action que constitue alors la notion de retour s'inscrit dans une perspective libérale, au sens économique et politique du terme. En effet, dans I'Italie giolittienne, la construction et le maniement des statistiques de retour ont eu un caractère plus comptable que proprement démographique : il s'agit bien alors de quantifier des flux de travailleurs en rotation, des flux de clients sur les navires, qu'on tente très vite de connecter avec l'évaluation des retours financiers. En outre, la production de cet instrument statistique repose à ce moment-là sur une coopération entre l'administration et les entreprises maritimes, et non sur un improbable maillage policier généralisé : les retours sont alors sortis d'une approche en termes de contrôle policier, pour être insérés dans des logiques comptables différentes. Par-là, on perçoit le risque d'erreur de perspective d'une historiographie qui a souvent eu tendance à rabattre tout contrôle et toute volonté de savoir sur les mouvements de personnes aux XIXe et XXe siècles sur des logiques et des instruments d'identification policiers ${ }^{30}$. Dans les années 1890-1914, la question du contrôle des migrants par les chiffres était déjà très clairement posée, mais avec des perspectives qui ressortaient tout autant, et sans doute davantage, aux logiques du libéralisme économique qu'au contrôle policier. On peut voir là

30 En ce sens, mon analyse pour les années 1890-1914 s'éloigne de celle qu'avance, pour la même période, Torpey (2005). 
une matrice historique, aujourd'hui trop souvent oubliée, des enjeux de luttes politiques et sociales du présent, où l'externalisation par les États et la " privatisation " des contrôles migratoires, ainsi que la légitimation du rôle déterminant donné aux entreprises privées dans l'économie du contrôle, sont perçues comme de radicales nouveautés ${ }^{31}$.

Un autre enjeu actuel du contrôle et de l'administration des mouvements migratoires par les chiffres trouve d'importantes résonances dans ce moment de fondation des statistiques de retours : au début du XXe siècle, les États concernés ont bien saisi qu'aucune politique migratoire ne peut se construire de manière isolée. Statisticiens, administrateurs, et économistes déterminés à trouver des instruments pertinents de mesure et d'action ont travaillé dans ce sens, notamment autour de I'Institut International de Statistique, pourtant la coordination de l'information statistique en matière migratoire reste un chantier difficile jusque dans les années 1920. C'est pourquoi la réalisation de statistiques migratoires internationales constitue un pilier majeur du projet de coordination des flux élaboré par le Bureau International du Travail dans I'entre-deux-guerres, dans la double optique qui est alors la sienne, de paix sociale et d'efficacité économique (Rosental, 2006).

\section{Références bibliographiques}

Balletta Francesco (1976) Le rimesse degli emigrati italiani e la bilancia dei pagamenti internazionali (1861-1974), Naples, Arte tipografica.

Bonelli Franco (1984) Alberto Beneduce, in Alberto Mortara Ed., I protagonisti dell'intervento pubblico in Italia, Milan, Franco Angeli, pp. 329-356.

Brian Éric (2002) Transactions statistiques au XIXe siècle. Mouvements internationaux de capitaux symboliques, Actes de la recherche en sciences sociales, 5 , pp. 34-46.

Brian Éric (1989) Statistique administrative et internationalisme statistique pendant la seconde moitié du XIXe siècle, Histoire et Mesure, 3-4, pp. 201-224.

Cerase Francesco Paolo (2001) L'onda di ritorno: i rimpatri, in Piero Bevilacqua, Andreina De Clementi e Emilio Franzina Eds., Storia dell'emigrazione italiana, vol. 1: Partenze, Turin, Donzelli, pp. 113-126.

Choate Mark (2008) Emigrant Nation. The Making of Italy Abroad, Cambridge (Mass.), Harvard University Press, 340 p.

Dakhlia Jocelyne (2013) Une Méditerranée entre deux mondes, ou des mondes continus, in Jocelyne Dakhlia et Wolfgang Kaiser Éds., Les Musulmans dans I'histoire de l'Europe, t. 2 : Passages et contacts en Méditerranée, Paris, Albin Michel, pp. 7-31.

Dakhlia Jocelyne (2011) Les musulmans en Europe occidentale au Moyen Âge et à l'époque moderne : une intégration invisible, in Jocelyne Dakhlia et Bernard Vincent Éds., Les Musulmans dans l'histoire de l'Europe, t. 1 : Une intégration invisible, Paris, Albin Michel, pp. 7-29.

31 Sur les évolutions les plus récentes, voir Rodier (2012). 
Daniels Roger (2004) Guarding the Golden Door. American Immigration Policy and Immigrants since 1882, New York, Hill and Wang, $344 \mathrm{p}$.

Desrosières Alain (2008) L'argument statistique, vol. 1 : Pour une sociologie historique de la quantification, Paris, Presses de l'École des Mines, 328 p.

Desrosières Alain (1993) La politique des grands nombres. Histoire de la raison statistique, Paris, La Découverte, 456 p.

Douki Caroline (2013, à paraître) The "Return Politics" of a Sending Country: The Italian Case, 1880s-1914, in Nancy L. Green and Roger Waldinger Eds., A Century of Transnationalism: Immigrants and their Homeland Connections, Urbana\&Chicago, University of Illinois Press.

Douki Caroline (2011) Protection sociale et mobilité transatlantique : les migrants italiens au début du XXe siècle, Annales. Histoire, Sciences sociales, 2, pp. 375-410.

Douki Caroline (2006) L'État libéral italien face à l'émigration de masse (18601914), in Nancy Green et François Weil Éds., Citoyenneté et émigration. Les politiques du départ, Paris, Les Éditions de I'EHESS, pp. 95-117.

Douki Caroline (2000) Les retombées financières de l'émigration et le développement régional en Italie entre XIXe et XXe siècle, in Gérard Chastagnaret Éd., Crise espagnole et nouveau siècle en Méditerranée. Politiques publiques et mutations structurelles des économies dans l'Europe méditerranéenne (fin XIXe-début XXe siècle), Aix-en-Provence, Casa de Velasquez-Publications de I'Université de Provence, pp. 131-145.

Douki Caroline (1994) Les maires de I'Italie libérale à l'épreuve de l'émigration : le cas des campagnes lucquoises, Mélanges de l'École Française de Rome-Italie et Méditerranée, 106, pp. 333-364.

Douki Caroline, Feldman David et Rosental Paul-André (2006) Pour une histoire relationnelle du ministère duTravail en France, en Italie et au Royaume-Uni dans I'entre-deux-guerres : le transnational, le bilatéral et l'interministériel en mațière de politique migratoire, in Alain Chatriot, Odile Join-Lambert et Vincent Viet Éds., Les politiques du Travail (1906-2006). Acteurs, institutions, réseaux, Rennes, Presses Universitaires de Rennes, pp. 143-159.

Ferretti Roberto (2003) The formation of a bureaucratic group between centre and periphery: engineers and local government in Italy from the liberal period to fascism (1861-1939), in Michèle Dagenais Ed., Municipal services and Employees in the Modern City. New Historic Approaches, Farnham/Burlington, Ashgate, pp. 66-83.

Feuerhahn Wolf et Rabault-Feuerhahn Pascale (Éds.) (2010) La fabrique internationale de la science : les congrès scientifiques de 1865 à 1945, Revue germanique internationale, 12.

Fontaine Laurence (2005) Montagnes et migrations de travail. Un essai de comparaison globale (XVe-XXe siècles), Revue d'histoire moderne et contemporaine, $52(2)$, pp. 26-48.

Franzina Emilio (1980) II «biometro delle nazioni». Primi rilevamenti sull'emigrazione, Quaderni storici, 15, pp. 966-1005.

Gagnon Marc-André (2000) Les réseaux de I'internationalisme statistique (18851914), in Jean-Pierre Beaud et Jean-Guy Prévost Éds, L'ère du chiffre. Systèmes statistiques et traditions nationales, Sainte-Foy, Presse de I'Université du Québec, pp. 189-220. 
Gardey Delphine (2008) Écrire, calculer, classer. Comment une révolution de papier a transformé les sociétés contemporaines (1800-1940), Paris, La Découverte, $319 \mathrm{p}$.

Garnieri Carlo (1995) L'ordine pubblico e la giustizia penale, in Raffaele Romanelli Ed., Storia dello Stato italiano dall'Unità a oggi, Rome, Donzelli, pp. 365-405.

Goody Jack (1979) La raison graphique. La domestication de la pensée sauvage, Paris, Les Éditions de Minuit, 272 p.

Grange Daniel (1994) L'Italie et la Méditerranée (1896-1911). Les fondements d'une politique étrangère, Rome, École Française de Rome, $1702 \mathrm{p}$.

Green Nancy (2002) Repenser les migrations, Paris, PUF, 138 p.

Green Nancy et Weil Françoịs (Éds.) (2006) Citoyenneté et émigration. Les politiques du départ, Paris, Les Éditions de l'EHESS, 274 p.

Harper Marjory (2010) The Homecoming Migrant, in Marjory Harper and Stephen Constantine, Migration and Empire, Oxford, Oxford University Press, pp. 306-337.

Jensen Richard (1989) Police Reform and Social Reform: Italy from the Crisis of the 1890s to the Giolittian Era, Criminal Justice History: An International Annual, vol. 10, Westport (Conn.)/Londres, Meckler, pp. 179-200.

Labbé Morgane (2003) Dénombrer les nationalités en Prusse au XIXe siècle : entre pratique d'administration locale et connaissance statistique de la population, Annales de démographie historique, 105, pp. 39-61.

Marucco Dora (2001) Le statistiche dell'emigrazione italiana, in Piero Bevilacqua, Andreina de Clementi e Emilio Franzina Eds., Storia dell'emigrazione italiana, vol. 1: Partenze, Turin, Donzelli, pp. 61-76.

Marucco Dora (1992) L'amministrazione della statistica italiana dall'Unità al fascismo, Turin, Pluriverso.

Massulo Gino (2001) Economia delle rimesse, in Piero Bevilacqua, Andreina de Clementi e Emilio Franzina Eds., Storia dell'emigrazione italiana, vol. 1: Partenze, Turin, Donzelli, pp. 161-183.

Melis Guido e Varni Angelo (Eds.) (1999) Burocrazie non burocratiche. Il lavoro dei technici nelle amministrazioni tra Otto e Novecento, Turin, Rosenberg \& Sellier.

Mittone Luigi (1984) Le rimesse degli emigrati sino al 1914, Affari sociali internazionali, 4, pp. 125-160.

Musi Aurelio (1998) Bureaucrazia comunale e mediazione politica nel Mezzogiorno tra Ottocento e Novecento, in Marco Soresina Ed., Colletti bianchi. Ricerche su impiegati, funzionari e technici in Italia fra '800 e '900, Milan, Franco Angeli, pp. 58-71.

Patriarca Silvana (1996) Numbers and Nationhood. Writing Statistics in Nineteenth-Century Italy, Cambridge, Cambridge University Press, 296 p.

Porter Ted (1995) Trust in Numbers. The Pursuit of Objectivity in Science and Public Life, Princeton, Princeton University Press, 324 p.

Prévost Jean-Guy (2009) A Total Science. Statistics in Liberal and Fascist Italy, Montreal, Mac Gill Queen's University Press, 335 p. 
Rodier Claire (2012) Xénophobie business. À quoi servent les contrôles migratoires?, Paris, La Découverte, $194 \mathrm{p}$.

Romanelli Raffaele (Ed.) (1995) Storia dello Stato italiano dall'Unità a oggi, Rome, Donzelli, 528 p.

Romanelli Raffaele (1989) Sulle carte interminate. Un ceto di impiegati tra privato e pubblico: $i$ secretari comunali in Italia, 1860-1915, Bologne, II Mulino.

Rosental Paul-André (2006) Géopolitique et État-Providence : le Bureau International du Travail et la politique mondiale des migrations dans I'entredeux-guerres, Annales. Histoire, Sciences sociales, 1, pp. 99-134.

Salvetti Patricia (1995) Immagine nazionale ed emigrazione nella Società "Dante Alighierin, Rome, Bonacci.

Soresina Marco (2001) Conoscere per amministrare. Luigi Bodio: statistica, economia e pubblica amministrazione, Milan, Franco Angeli.

Thévenot Laurent (2006) L'action au pluriel. Sociologie des régimes d'engagement, Paris, La Découverte, pp. 141-147.

Torpey John (2005) L'invention du passeport. États, citoyenneté et surveillance, Paris, Belin, 255 p.

Tosatti Giovanna (2009) Storia del Ministero dell'Interno. Dall'Unità alla regionalizzazione, Milan, II Mulino, 339 p.

Vatin François (Éd.) (2009) Évaluer et valoriser : une sociologie économique de la mesure, Toulouse, Presses Universitaires du Mirail, 306 p.

Wyman Mark (2005) Emigrants returning: the evolution of a tradition, in Marjory Harper Ed., Emigrant Homecomings. The Return Movement of Emigrants, 1600 2000, Manchester, Manchester University Press, pp. 16-31.

Wyman Mark (1993) Round-Trip to America. The Immigrants Return to Europe, 1880-1930, Ithaca, Cornell University Press, 272 p.

Zimmermann Bénédicte (1994) Statisticiens des villes allemandes et action réformatrice (1871-1914). La construction d'une généralité statistique, Genèses, 15, pp. 4-27. 


\section{Caroline Douki}

\section{Compter les « retours " d'émigrants dans I'ltalie du début du $\mathrm{XXe}$ siècle : conventions statistiques, libéralisme économique et politique publique}

Cet article propose de restituer, à partir de ce qui se déroule dans les sphères savantes et administratives italiennes au cours des années 1870-1920, le caractère graduel du processus par lequel le " retour " est devenu une catégorie statistique bien individualisée, en relation avec des questions cognitives mais aussi techniques, administratives et politiques. L'un des grands enjeux attachés à la présentation statistique du phénomène des retours résidait déjà dans les possibilités d'utiliser ces chiffres dans le cadre de politiques publiques destinées à encadrer les migrations et à soutenir le développement économique. Bien que l'État italien fût alors l'un des plus précocement mobilisés sur cette question, l'organisation du dispositif de relevé, de classification et de calcul des flux de retours y a rencontré de nombreuses difficultés. La configuration italienne est d'autant plus instructive, qu'elle s'articule étroitement aux institutions et aux débats statistiques, économiques, démographiques internationaux de l'époque.

\section{Counting Emigrants" "Returns" to Italy at the Beginning of the 20th Century: Statistical Conventions, Economic Liberalism and Public Policy}

On the basis of what takes place in Italian academic and administrative spheres between 1870 and 1920, this article proposes a restitution of the gradual nature of the process by which "return" became an extremely individualized statistical category in conjunction with cognitive, but also technical, administrative, and political questions. One of the major issues related to the statistical presentation of the phenomenon of returns was already present in the very possibility of using these numbers in the context of public policies meant to give a framework to migrations and to lend support to economic development. Even though the Italian State was one of the first to mobilize around this issue, the organization of the system that allowed for the observation, classification, and calculation of currents of return encountered many difficulties. The Italian configuration is thus all the more instructive since it is closely articulated with its institutions and with international debates on statistics, economics, and demographics from that time.

\section{Contabilizar los retornos de emigrantes en Italia al comienzo del siglo XX: convenciones estadísticas, liberalismo económico y políticas públicas}

El artículo propone una restitución, a partir de lo ocurrido en las esferas eruditas y administrativas italianas durante los años 1870-1920, del carácter gradual del proceso a partir del cual el "retorno" sobrevino una categoría estadística individualizada, todo ello en relación con cuestiones cognitivas, técnicas, administrativas y políticas. Uno de los grandes aspectos ligados a la presentación estadística del fenómeno del "retorno" residía en la posibilidad de utilizar las cifras en el marco de políticas públicas destinadas a regular las migraciones y a apoyar el desarrollo económico. A pesar de que el Estado italiano fue uno de los pioneros en esta cuestión, la organización de un dispositivo de recuento, de clasificación y de cálculo de los flujos de retornos hizo frente a numerosas dificultades. La configuración italiana de este proceso es sumamente instructiva debido a su articulación estrecha con las instituciones y los debates estadísticos, económicos y demográficos a nivel internacional que tuvieron lugar en aquella época. 